\title{
Caracterización de las especies de mangle ubicadas en inmediaciones de la escuela naval de cadetes "Almirante Padilla"
}

\author{
Cristhian Mendoza Pérez ${ }^{1}$ \\ Marena Vitola Quintero ${ }^{2}$ \\ TK Luis López Osorio ${ }^{3}$ \\ TK Johan Rodríguez Ortiz ${ }^{4}$
}

\section{Resumen}

En el presente trabajo está consignada la caracterización de las especies de manglar que se encuentran presentes en la Isla de Manzanillo, así como un análisis sobre la distribución de las mismas en el área de estudio. Cabe mencionar, que este ecosistema es ampliamente biodiverso e importante para el medio ambiente marino y terrestre, pero debido al efecto antrópico acentuado producto de diferentes actividades en la isla, sus condiciones no son las mejores. Tanto la fauna como la flora del bosque de manglar se ven afectados cuando los factores bióticos o abióticos cambian, ya sea por acción humana o situaciones naturales, es por esto, que en el marco del presente trabajo se plasmaron las bondades del ecosistema para el medio ambiente y la comunidad en general, lo cual lo convierte en objeto de preservación y uso sostenible para la Armada Nacional, de acuerdo a lo consignado en sus ejes de investigación más específicamente "Intereses Marítimos", debido a esto, la investigación se centró en identificar las especies de manglar presentes en los alrededores de la Isla de Manzanillo y su distribución. Lo anterior a través de muestreo en campo según la metodología de Ulloa-Delgado et al. (1998), así como el cálculo y análisis del Índice de Valor de Importancia de Especies de Curtis $\&$ Mcintosh (1950).

\section{Palabras Claves}

Manglar, ecosistema, intereses marítimos, caracterización, Isla de Manzanillo

\begin{abstract}
In the present work, the characterization of the mangrove species that are present in the Island of Manzanillo is included, as well as an analysis of the distribution of the same in the study area. It is worth mentioning that this ecosystem is widely biodiverse and important for the marine and terrestrial environment, but due to the anthropic effect accentuated by different activities on the island, its conditions are not the best. Both the fauna and the flora of the mangrove forest are affected when the biotic or abiotic factors change, either by human action or natural situations, that is why, in the framework of this work the benefits of the ecosystem for the environment were reflected environment and the community in general, which makes it an object of preservation and sustainable use for the National Navy, according to what is stated in its research lines more specifically "Maritime Interests", due to this, the investigation focused on identifying the mangrove species present around the Isle of Manzanillo and its distribution. The above through field sampling according to the methodology of Ulloa-Delgado et al. (1998), as well as the calculation and analysis of the Curtis \& Mcintosh Species Importance Value Index (1950).
\end{abstract}

\section{Keywords}

Mangrove, ecosystem, maritime interests, characterization, Isle of Manzanillo.

Fecha de recepción: 1 de Mayo de 2019, Fecha de evaluación: 15 de Mayo de 2019, Fecha de aceptación: 5 Junio de 2019

\footnotetext{
1. Magister en biología celular y estructural. Biólogo. Facultad de Ciencias Navales Escuela Naval de Cadetes "Almirante Padilla". Correo electrónico: poseidon@enap.edu.co

2. Magister en Diseño, Gestión y Dirección de Proyectos. Especialización en Telecomunicaciones. Ingeniera de Sistemas. Facultad de Ciencias Navales Escuela Naval de Cadetes "Almirante Padilla". Correo electrónico: jpcna@enap.edu.co

3. Profesional en Ciencias Navales. Facultad de Ciencias Navales Escuela Naval de Cadetes "Almirante Padilla". Correo electrónico: luis.lopez@armada.mil.co

4. Profesional en Ciencias Navales. Facultad de Ciencias Navales Escuela Naval de Cadetes "Almirante Padilla". Correo electrónico: Johan.rodriguez@armada.mil.co
} 


\section{Introducción}

Los manglares son ecosistemas vitales para la biodiversidad por ser áreas de protección para los primeros estadios de vida de los recursos hidrobiológicos; porque aportan nutrientes al medio marino que constituyen la base de la productividad primaria fundamental en la cadena alimenticia del océano; porque son básicos para la conservación de la línea litoral, ya que evitan la erosión que producen las corrientes y las olas que golpean la costa; y porque cumplen una función filtradora de las cargas orgánicas provenientes de fuentes terrestres, que en la ausencia de este recurso causarían graves perjuicios sobre la vida marina (Ministerio de Medio Ambiente, 1995).

Actualmente la Escuela Naval de Cadetes "Almirante Padilla" (ENAP) cuenta con una amplia extensión de bosque de mangle que cubre gran parte del área costera de la Isla de Manzanillo, la cual comprende desde la Escuela de Guardacostas hasta la zona conocida como Punta Recluta, área ubicada al extremo sur de la isla. Este ecosistema presenta un deterioro medioambiental, debido al efecto antrópico al que se encuentra sometido de manera constante, ejemplo de ello son las aguas residuales vertidas directamente al cuerpo de agua por la comunidad aledaña, así como, las basuras solidas que van a parar a las raíces de estos árboles generando un impacto negativo tanto a la fauna como a la flora lugareña.

Además de lo anterior, dentro de esos efectos antrópicos se puede encontrar la sobreexplotación de la madera de este tipo de ecosistema, que se caracteriza por ser de una excelente calidad para la construcción casas y la producción de carbón. Sin embargo, la tala es en raras ocasiones la principal causa de la pérdida de estos árboles, existen otros factores tales como la competencia por la tierra para desarrollos urbanos, turismo, agricultura o construcción de estanques para cultivo de camarón. La gran tasa de cambios negativos en los manglares en los años ochenta en Asia, el Caribe y Latinoamérica ha sido ocasionada principalmente por la conversión de estas áreas para acuicultura e infraestructura, ya que muchos gobiernos han optado por ella con la intención de aumentar la seguridad alimenticia, estimular las economías nacionales y mejorar los estándares de vida (Calderón, Aburto \& Ezcurra, 2009)

Con base en lo descrito, el presente proyecto permitió identificar las especies de manglar presentes en la ENAP, así como su distribución en el área en donde se encuentran, información que sirve como base para que a futuro se puedan formular estrategias encaminadas a la conservación de este valioso ecosistema el cual hace parte de los intereses marítimos de la nación y es una prioridad para la Armada Nacional, como se refleja en la línea de investigación "Desarrollo Institucional Sostenible, Poder Marítimo y Educación Militar" y la sub-línea "Seguridad y Sostenibilidad Ecológica, Económica y Ambiental" las cuales hacen parte del grupo de investigación POSEIDÓN adscrito a la Facultad de Ciencias Navales y Náuticas (Escuela Naval de Cadetes "Almirante Padi1la”, 2016)

\section{Metodología}

Para determinar la estructura del manglar presente en la ENAP, se establecieron 2 estaciones de muestreo, en cada estación se situó un transecto lineal perpendicular a la línea de costa con ayuda de una cinta métrica. La longitud de los transectos varió de acuerdo con la extensión de la franja de manglar presente en cada estación (Aproximadamente entre $25 \mathrm{~m}$ y $50 \mathrm{~m}$ ). Para el caso de la estación de muestreo 1 a lo largo del transecto se delimitaron tres parcelas de 10×10 metros a través del uso cuerda, separadas entre sí por una distancia de 10m (Figura 1), para el caso de la estación de muestreo 2 se delimitaron tres parcelas de $5 \times 5$ metros separadas a su vez por una distancia de $5 \mathrm{~m}$ (Figura 2) (basado en Ulloa-Delgado et al., 1998).

De la misma manera estos transectos se tomaron a una distancia de la línea de costa proporcional al tamaño de cada parcela, es decir, en la zona 1 , cuyas parcelas fueron de $10 \mathrm{~m}$ x $10 \mathrm{~m}$ se tomó una distancia de $10 \mathrm{~m}$ respecto a la línea de costa. Situación similar se realizó con la zona 2. 


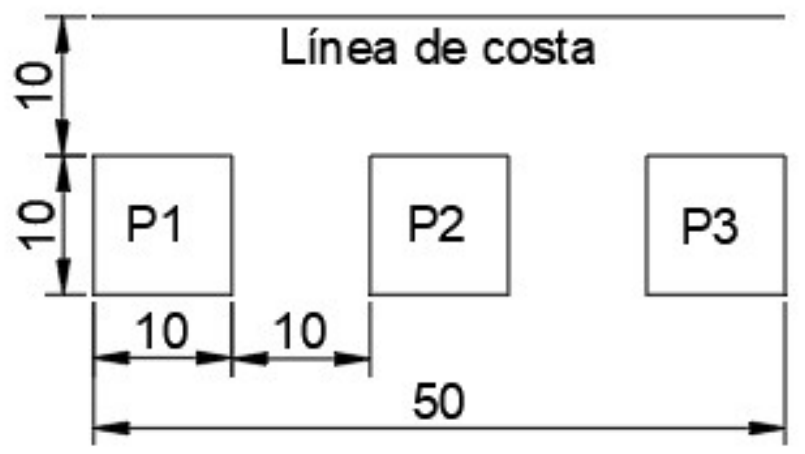

Figura 1. Esquema del transecto utilizado en el levantamiento de muestras por parcelas de $(10 \mathrm{~m} \times 10 \mathrm{~m})$ en la estación de muestreo 1.

Fuente: Elaboración Propia

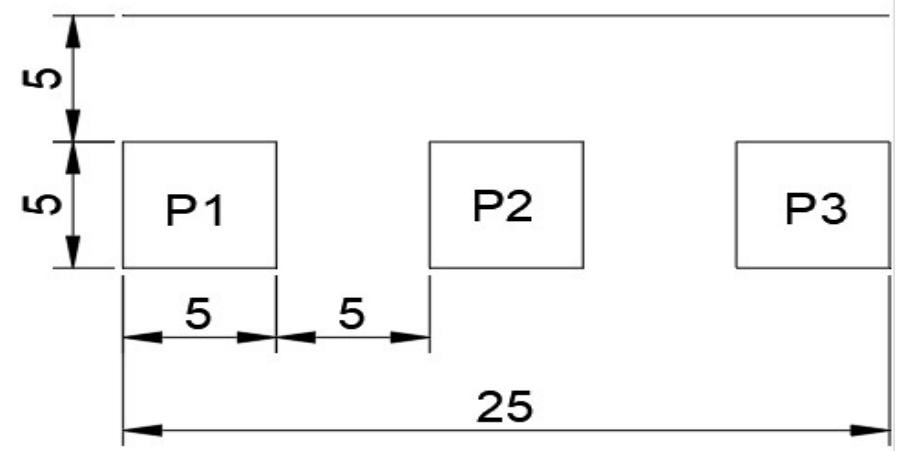

Figura 2. Esquema del transecto utilizado en el levantamiento de muestras por parcelas de $(5 \mathrm{~m} \times 5 \mathrm{~m})$ en la estación de muestreo 2.

Fuente: Elaboración Propia

Las zonas de muestreo se escogieron teniendo en cuenta la distribución que presenta el bosque de manglar en inmediaciones de la Escuela Naval de Cadetes "Almirante Padilla" siendo identificada la zona de muestreo 1 con el nombre "Muelle ENAP" y la zona de muestreo 2 como "Punta
Recluta" (Figura 3). Cabe mencionar que el perímetro de cada parcela se delimitó con una cuerda para tener claro cuáles eran los individuos que se encontraban dentro de la zona delimitada, así mismo, se procedió a contabilizar los individuos por especie.

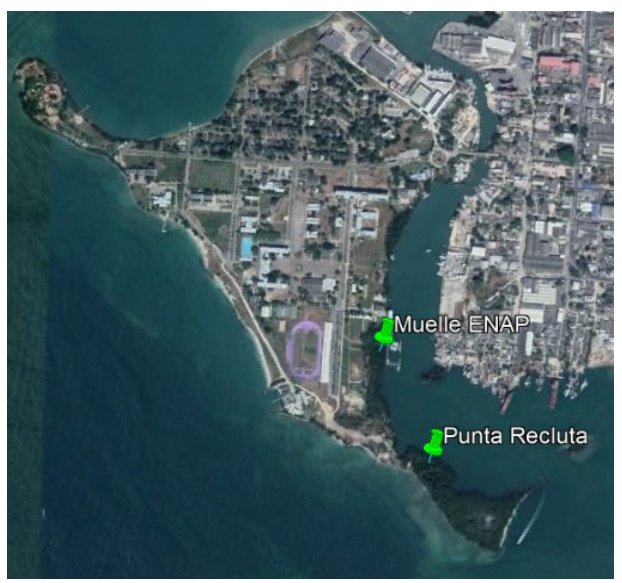

Figura 3. Isla de Manzanillo con zonas de muestreo indicadas

Fuente: Imagen tomada con Google Earth (2018) 
Posteriormente se realizó un conteo e identificación de todos los individuos y especies de mangle encontradas en las parcelas de cada estación a través de anotaciones de campo de las características morfológicas que después sirvieron de apoyo en la identificación de especies basada en revisión de literatura; cabe resaltar que a cada individuo de mangle se le tomaron medidas de la circunferencia a la altura del pecho (CAP) con ayuda de una cinta métrica flexible; con base en este dato se calculó el diámetro a la altura del pecho (DAP), teniendo en cuenta sugerencia de Catie (1994, citado en Quiceno, Tangarife \& Álvarez, 2015) (Figura 4).

$$
D A P=\frac{C A P}{\pi} * \frac{1 \mathrm{~cm}}{10 \mathrm{~mm}}
$$

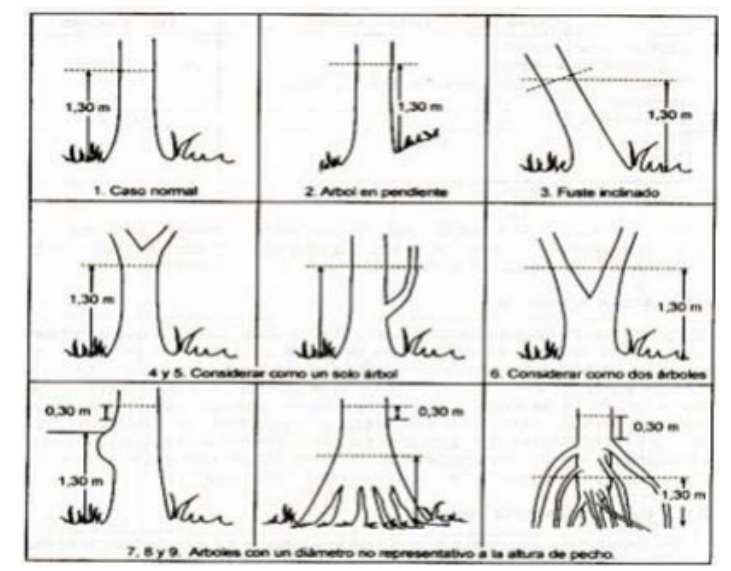

Figura 4. Recomendaciones para medir el DAP de un árbol según CATIE (1994)

Fuente: Quiceno, Tangarife \& Álvarez (2015)

Con los datos obtenidos del levantamiento de muestras se calculó el IVI (Índice de Valor de Importancia), por medio del cálculo de Abundancia Relativa, Área Basal, Dominancia Relativa y Frecuencia Relativa de los individuos y de las especies de mangle de la zona de muestreo (Curtis \& Mcintosh, 1951) a través de las siguientes formulas:

La abundancia es la proporción de individuos de una especie entre el total de individuos en una zona de muestreo.

Abundancia Relativa $\left(A_{i} \%\right): \frac{\text { Número de individuos de la especie }}{\text { Número total de individuos }} * 100 \%$

El área basal es la superficie de una sección transversal del tronco del individuo a la altura del pecho (1.3m de altura). Es un parámetro muy preciso. No hay solapamiento. Se expresa en $\mathrm{m} 2$.$$
\text { Área Basal (De un individuo): DAP } * \frac{\pi}{4}
$$$$
\text { Área Basal de una especie: } \sum A B_{\text {De los inaividuos de la misma especie }}
$$

La dominancia en ecología representa la impor- tancia de una especie en función de su desarrollo o biomasa. En bosques se mide a través del área basal. La Dominancia Absoluta (Di) es la suma de área basal de todos los individuos de una especie. La Dominancia Relativa (Di\%) es la relación porcentual entre la dominancia absoluta de una especie con respecto al área basal total de la parcela.

Dominancia Relativa $\left(D_{i} \%\right): \frac{\text { Área Basal por Especie }}{\text { Área Basal Total }} * 100 \%$

La frecuencia es la probabilidad de encontrar una especie en una unidad muestral particular, o en este caso en las parcelas.

Frecuencia Relativa $\left(F_{i} \%\right): \frac{\text { Parcelas en las que se presenta la especie }}{\text { Parcelas totales tomadas }} * 100 \%$

Una vez con estos datos fue posible calcular el IVI (Índice de Valor de Importancia) de cada una de las especies.

Índice de Valor de Importancia (IVI): $A_{i} \%+D_{i} \%+F_{i} \%$

Índice de Valor de Importancia \%(IVI\%): $\frac{I V I_{\text {de la especie }}}{I V I_{\text {Total }}} \%$ 
Para poder consignar todos los presentes datos se construyeron formatos de tablas, que permitieron organizar y consignar los datos de cantidad de in- dividuos, CAP de los individuos, y finalmente un formato para plasmar todos los cálculos para el Índice de Valor de Importancia.

\section{Resultado y discusión}

Para el caso de la Zona del Muelle ENAP, para efectos de geo posicionamiento se registraron las coordenadas Latitud $10^{\circ} 23^{\prime} 12.23$ ' N y Longitud $-75^{\circ} 31^{\prime} 38.58^{\prime \prime}$ O, una vez aplicado el procedimiento y técnicas descritas en la metodología se procedió a tabular la información recopilada como se muestra en la Tabla 1, donde se observa el número de especies por parcela, visualizándose cla- ramente un predominio de Rizophora mangle en todas las parcelas, teniendo como característica morfológica más importante sus sus raíces en forma de zancos, poseen hojas simples, opuestas, pecioladas, de hoja redondeada, elípticas a oblongas, estas se aglomeran en las puntas de las ramas, su color es verde oscuro en el haz y amarillentas en el envés (Olguín, Hernández y Sanchez-Galván, 2007)

Tabla 1. Zona Muelle ENAP

\begin{tabular}{|l|l|l|l|}
\hline \multicolumn{1}{|c|}{ Especie } & Parcela 1 & Parcela 2 & Parcela 3 \\
\hline Rhizophora mangle & 13 & 14 & 12 \\
\hline Laguncularia Racemosa & 1 & 2 & 1 \\
\hline Conocarpus erectus & 2 & 2 & 2 \\
\hline Total & 16 & 18 & 15 \\
\hline
\end{tabular}

Nota. El número total de individuos en esta zona fue de 49 , entre las tres especies. Se nota un claro predominio de Rhizophora mangle en esta zona.

Fuente: Elaboración propia basado en Cortés (2010).

En segunda instancia se recopiló la información de la toma de muestras, el Diámetro a la Altura en detalle de todos los individuos encontrados en del Pecho (DAP) obtenido con base en la medida las parcelas de la Zona de Muestreo 1. Cada uno anterior y el área basal de cada individuo (AB) lleva referenciados los datos de Circunferencia a (Tabla 2): la Altura del Pecho (CAP) tomados al momento

Tabla 2. Levantamiento de muestras Zona Muelle ENAP

\begin{tabular}{|l|l|l|l|l|l|l|l|}
\hline $\mathbf{P}$ & Sp & Num & Nombre Vulgar & \multicolumn{1}{|c|}{ Nombre Cientifico } & CAP(mm) & DAP(cm) & AB(m2) \\
\hline 1 & 1 & 1 & Mangle Rojo & Rhizophora Mangle & 520 & 16,55 & 0,022 \\
\hline 1 & 1 & 2 & Mangle Rojo & Rhizophora Mangle & 486 & 15,47 & 0,019 \\
\hline 1 & 1 & 3 & Mangle Rojo & Rhizophora Mangle & 531 & 16,9 & 0,022 \\
\hline 1 & 1 & 4 & Mangle Rojo & Rhizophora Mangle & 513 & 16,33 & 0,021 \\
\hline 1 & 1 & 5 & Mangle Rojo & Rhizophora Mangle & 485 & 15,44 & 0,019 \\
\hline 1 & 1 & 6 & Mangle Rojo & Rhizophora Mangle & 465 & 14,8 & 0,017 \\
\hline 1 & 1 & 7 & Mangle Rojo & Rhizophora Mangle & 476 & 15,15 & 0,018 \\
\hline 1 & 1 & 8 & Mangle Rojo & Rhizophora Mangle & 495 & 15,76 & 0,019 \\
\hline 1 & 1 & 9 & Mangle Rojo & Rhizophora Mangle & 514 & 16,36 & 0,021 \\
\hline 1 & 1 & 10 & Mangle Rojo & Rhizophora Mangle & 448 & 14,26 & 0,016 \\
\hline 1 & 1 & 11 & Mangle Rojo & Rhizophora Mangle & 495 & 15,76 & 0,019 \\
\hline 1 & 1 & 12 & Mangle Rojo & Rhizophora Mangle & 524 & 16,68 & 0,022 \\
\hline 1 & 1 & 13 & Mangle Rojo & Rhizophora Mangle & 508 & 16,17 & 0,021 \\
\hline
\end{tabular}


Cristhian Mendoza Pérez, Marena Vitola Quintero, TK Luis López Osorio, TK Johan Rodríguez Ortiz

\begin{tabular}{|c|c|c|c|c|c|c|c|}
\hline $\mathbf{P}$ & Sp & Num & Nombre Vulgar & Nombre Cientifico & CAP(mm) & DAP(cm) & $\mathrm{AB}(\mathrm{m} 2)$ \\
\hline 1 & 2 & 14 & Mangle Blanco & Laguncularia Racemosa & 423 & 13,46 & 0,014 \\
\hline 1 & 3 & 15 & Mangle Zaragoza & Conocarpus erectus & 622 & 19,8 & 0,031 \\
\hline 1 & 3 & 16 & Mangle Zaragoza & Conocarpus erectus & 594 & 18,91 & 0,028 \\
\hline 2 & 1 & 17 & Mangle Rojo & Rhizophora Mangle & 527 & 16,77 & 0,022 \\
\hline 2 & 1 & 18 & Mangle Rojo & Rhizophora Mangle & 530 & 16,87 & 0,022 \\
\hline 2 & 1 & 19 & Mangle Rojo & Rhizophora Mangle & 503 & 16,01 & 0,02 \\
\hline 2 & 1 & 20 & Mangle Rojo & Rhizophora Mangle & 497 & 15,82 & 0,02 \\
\hline 2 & 1 & 21 & Mangle Rojo & Rhizophora Mangle & 499 & 15,88 & 0,02 \\
\hline 2 & 1 & 22 & Mangle Rojo & Rhizophora Mangle & 526 & 16,74 & 0,022 \\
\hline 2 & 1 & 23 & Mangle Rojo & Rhizophora Mangle & 431 & 13,72 & 0,015 \\
\hline 2 & 1 & 24 & Mangle Rojo & Rhizophora Mangle & 514 & 16,36 & 0,021 \\
\hline 2 & 1 & 25 & Mangle Rojo & Rhizophora Mangle & 508 & 16,17 & 0,021 \\
\hline 2 & 1 & 26 & Mangle Rojo & Rhizophora Mangle & 481 & 15,31 & 0,018 \\
\hline 2 & 1 & 27 & Mangle Rojo & Rhizophora Mangle & 456 & 14,51 & 0,017 \\
\hline 2 & 1 & 28 & Mangle Rojo & Rhizophora Mangle & 476 & 15,15 & 0,018 \\
\hline 2 & 1 & 29 & Mangle Rojo & Rhizophora Mangle & 526 & 16,74 & 0,022 \\
\hline 2 & 1 & 30 & Mangle Rojo & Rhizophora Mangle & 476 & 15,15 & 0,018 \\
\hline 2 & 2 & 31 & Mangle Blanco & Laguncularia Racemosa & 456 & 14,51 & 0,017 \\
\hline 2 & 2 & 32 & Mangle Blanco & Laguncularia Racemosa & 432 & 13,75 & 0,015 \\
\hline 2 & 3 & 33 & Mangle Zaragoza & Conocarpus erectus & 616 & 19,61 & 0,03 \\
\hline 2 & 3 & 34 & Mangle Zaragoza & Conocarpus erectus & 605 & 19,26 & 0,029 \\
\hline 3 & 1 & 35 & Mangle Rojo & Rhizophora Mangle & 510 & 16,23 & 0,021 \\
\hline 3 & 1 & 36 & Mangle Rojo & Rhizophora Mangle & 487 & 15,5 & 0,019 \\
\hline 3 & 1 & 37 & Mangle Rojo & Rhizophora Mangle & 477 & 15,18 & 0,018 \\
\hline 3 & 1 & 38 & Mangle Rojo & Rhizophora Mangle & 418 & 13,31 & 0,014 \\
\hline 3 & 1 & 39 & Mangle Rojo & Rhizophora Mangle & 531 & 16,9 & 0,022 \\
\hline 3 & 1 & 40 & Mangle Rojo & Rhizophora Mangle & 471 & 14,99 & 0,018 \\
\hline 3 & 1 & 41 & Mangle Rojo & Rhizophora Mangle & 482 & 15,34 & 0,018 \\
\hline 3 & 1 & 42 & Mangle Rojo & Rhizophora Mangle & 471 & 14,99 & 0,018 \\
\hline 3 & 1 & 43 & Mangle Rojo & Rhizophora Mangle & 514 & 16,36 & 0,021 \\
\hline 3 & 1 & 44 & Mangle Rojo & Rhizophora Mangle & 516 & 16,42 & 0,021 \\
\hline 3 & 1 & 45 & Mangle Rojo & Rhizophora Mangle & 524 & 16,68 & 0,022 \\
\hline 3 & 1 & 46 & Mangle Rojo & Rhizophora Mangle & 529 & 16,84 & 0,022 \\
\hline 3 & 2 & 47 & Mangle Blanco & Laguncularia Racemosa & 448 & 14,26 & 0,016 \\
\hline 3 & 3 & 48 & Mangle Zaragoza & Conocarpus erectus & 584 & 18,59 & 0,027 \\
\hline 3 & 3 & 49 & Mangle Zaragoza & Conocarpus erectus & 596 & 18,97 & 0,028 \\
\hline
\end{tabular}

Nota. Se evidencian los datos de cada uno de los mangles encontrados en esta zona, se denota claramente la prevalencia del Rhizophora mangle en la misma. En la tabla se referencian P como el número de parcela, Sp como numero asignado a cada especie y Num como el número de cada individuo en cada parcela.

Fuente: Elaboración propia basado en Cortés (2010).

De acuerdo a los plasmado en las Tablas 1 y 2, es bastante claro el predominio del mangle rojo por encima de las otras dos especies registradas, esto puede deberse a que se trata de una especia pione-

ra y se encuentra en mayor cantidad con relación a las restantes (Ecured, 2018), lo que indica que su papel ecológico consiste en iniciar la sucesión para que especies como mangle blanco (que pre- 
sentó hojas de forma ovadas o elípticas con ápice agudo o acuminado, así como una corteza clara y glándulas excretoras de sal en la base de la hoja, según lo descrito por Olguín, Hernández, \& Sánchez, 2007) y mangle negro (que presentó corteza obscura y cuyo carácter morfológico principal en campo fue la presencia de neumatóforos de acuerdo a lo descrito por Mejía, Molina, Grijalba, $\&$ Niño, 2014) puedan establecerse y reproducirse en condiciones favorables, además Rizhophora mangle presenta una alta tolerancia a la salinidad congruente con las características del bosque de manglar en el mar Caribe, según lo descrito por Prahl (1990). Cabe resaltar que los diámetros aquí plasmados van desde los $13,31 \mathrm{~cm}$ reporta- do para mangle rojo hasta $19.8 \mathrm{~cm}$ para mangle zaragoza valores de DAP que se encuentran dentro del rango de lo reportado por Cortés (2010) para las misma especies, las cuales van desde 2,5 $\mathrm{cm}$ hasta $87 \mathrm{~cm}$, esta especie se caracterizó por presentar una corteza de color ceniza o café y un fruto en forma de pequeña piña (Jacome, 2006).

De otro lado en la Tabla 3 se presenta el cálculo de la Abundancia Relativa ( $\mathrm{A}_{\mathrm{i}} \%$ ), la Dominancia Relativa $\left(\mathrm{D}_{\mathrm{i}} \%\right)$ y la Frecuencia Relativa $\left(\mathrm{F}_{\mathrm{i}} \%\right)$, y con esto hallar el Índice de Valor de Importancia (IVI) y el Índice de Valor de Importancia Relativo (IVI\%) para las especies reportadas en la primera estación de muestreo:

Tabla 3. Cálculo de IVI en la zona Muelle ENAP

\begin{tabular}{|l|l|l|l|l|l|}
\hline \multicolumn{1}{|c|}{ Especie } & \multicolumn{1}{|c|}{$\mathbf{A i} \%$} & \multicolumn{1}{c|}{$\mathbf{D i} \%$} & \multicolumn{1}{c|}{$\mathbf{F i} \%$} & \multicolumn{1}{c|}{ IVI } & \multicolumn{1}{c|}{ IVI\% } \\
\hline Rhizophora mangle & $79,59 \%$ & $76,50 \%$ & $33,33 \%$ & $189,42 \%$ & $63,14 \%$ \\
\hline Laguncularia Racemosa & $8,16 \%$ & $6,16 \%$ & $33,33 \%$ & $47,65 \%$ & $15,88 \%$ \\
\hline Conocarpus erectus & $12,24 \%$ & $17,35 \%$ & $33,33 \%$ & $62,92 \%$ & $20,97 \%$ \\
\hline
\end{tabular}

Nota. El Rhizophora mangle representa aproxidamante la mitad de la importancia en la zona de muestreo 1 , con un IVI\% del 63,14\%. Se puede observar que la relación de valores en el calculo de IVI de abundancia y dominancia son proporcionales en cierta medida, es posible inferir que ambos datos confirman el analisis expuesto, puesto que la proporcion mucho mayor de individuos de Rhizophora mangle presentan mucha mas biomasa que las demas especies.

Fuente: Elaboración propia basado en Cortés (2010).

Con base en lo ilustrado en la Tabla 3, el IVI de la especie Rizophora mangle $(63,14 \%)$ es muy superior al de las especies de Laguncularia racemosa $(15,88 \%)$ y Conocarpus erectus $(20,97 \%)$, lo que quiere decir en primera instancia, que en términos de presencia el mangle rojo se registra con mayoría de individuos en cada una de las parcelas pertenecientes a este transecto, sin embargo a pesar de esto, el valor de la Frecuencia Relativa fue el mismo con respecto al mangle blanco y el mangle zaragoza $(33,33)$, esto se puede explicar debido a que para el cálculo de la Frecuencia Relativa se tiene en cuenta el porcentaje de aparición de la especie sobre el total de parcelas, lo que indica que en las tres parcelas hay presencia de las tres especies, por lo tanto la distribución de las mismas es bastante amplia a lo largo del área objeto de estudio, pero con número diferente de individuos por parcela; de otro lado la
Abundancia Relativa del mangle rojo presentó un valor de 79,59 valor mayor al obtenido para mangle blanco $(8,16)$ y mangle zaragoza $(12,24)$, lo que indica que el número de individuos de mangle rojo es superior a las otras especies muestreadas respecto a la unidad de superficie que comprende cada una de las parcelas; finalmente en el caso de la Dominancia Relativa el mangle rojo obtuvo un $76,50 \%$ siendo como en el caso de las otras medidas superior al resto de especies, lo que se traduce en que la suma de las áreas del tronco determinadas a la altura del pecho es mayor en el caso del mangle rojo, significando que esta especie está mejor adaptada a las condiciones actuales del ecosistema ya que constituye el mayor aporte de biomasa al mismo, es decir el mayor volumen de madera lo aporta dicha especie, el anterior análisis se realizó con base en Curtis y McIntosh (1951). 
Cristhian Mendoza Pérez, Marena Vitola Quintero, TK Luis López Osorio, TK Johan Rodríguez Ortiz

De otro lado, a continuación se presentan los tud -75³1'34.61' O. La información obtenida resultados obtenidos de la zona de muestreo 2, producto de la toma de información en la zona "Punta Recluta", la cual está ubicada en las si- de muestreo 2, se consigna en las Tablas 4 y 5. guientes coordenadas $10^{\circ} 23^{\prime} 2.24^{\prime \prime} \mathrm{N}$ y Longi-

Tabla 4. Zona Punta Recluta

\begin{tabular}{|l|l|l|l|}
\hline \multicolumn{1}{|c|}{ Especie } & Parcela 1 & Parcela 2 & Parcela 3 \\
\hline Rhizophora mangle & 7 & 5 & 4 \\
\hline Laguncularia Racemosa & 0 & 1 & 0 \\
\hline Total & 7 & 6 & 4 \\
\hline
\end{tabular}

Nota. El número total de individuos en esta zona fue de 17, entre las tres especies. Se nota un claro predominio de Rhizophora mangle en esta zona.

Fuente: Elaboración propia basado en Cortés (2010).

Tabla 5. Levantamiento de muestras zona Punta Recluta

\begin{tabular}{|l|l|l|l|l|l|l|l|}
\hline $\mathbf{P}$ & Sp & Num & Nombre Vulgar & Nombre Científico & CAP(mm) & DAP(cm) & AB(m2) \\
\hline 1 & 1 & 1 & Mangle Rojo & Rhizophora mangle & 406 & 12,92 & 0,013 \\
\hline 1 & 1 & 2 & Mangle Rojo & Rhizophora mangle & 260 & 8,28 & 0,005 \\
\hline 1 & 1 & 3 & Mangle Rojo & Rhizophora mangle & 383 & 12,19 & 0,012 \\
\hline 1 & 1 & 4 & Mangle Rojo & Rhizophora mangle & 381 & 12,13 & 0,012 \\
\hline 1 & 1 & 5 & Mangle Rojo & Rhizophora mangle & 477 & 15,18 & 0,018 \\
\hline 1 & 1 & 6 & Mangle Rojo & Rhizophora mangle & 436 & 13,88 & 0,015 \\
\hline 1 & 1 & 7 & Mangle Rojo & Rhizophora mangle & 388 & 12,35 & 0,012 \\
\hline 2 & 1 & 8 & Mangle Rojo & Rhizophora mangle & 415 & 13,21 & 0,014 \\
\hline 2 & 1 & 9 & Mangle Rojo & Rhizophora mangle & 443 & 14,1 & 0,016 \\
\hline 2 & 1 & 10 & Mangle Rojo & Rhizophora mangle & 412 & 13,11 & 0,014 \\
\hline 2 & 1 & 11 & Mangle Rojo & Rhizophora mangle & 395 & 12,57 & 0,012 \\
\hline 2 & 1 & 12 & Mangle Rojo & Rhizophora mangle & 378 & 12,03 & 0,011 \\
\hline 2 & 2 & 13 & Mangle Blanco & Laguncularia Racemosa & 421 & 13,4 & 0,014 \\
\hline 3 & 1 & 14 & Mangle Rojo & Rhizophora mangle & 368 & 11,71 & 0,011 \\
\hline 3 & 1 & 15 & Mangle Rojo & Rhizophora mangle & 377 & 12 & 0,011 \\
\hline 3 & 1 & 16 & Mangle Rojo & Rhizophora mangle & 412 & 13,11 & 0,014 \\
\hline 3 & 1 & 17 & Mangle Rojo & Rhizophora mangle & 406 & 12,92 & 0,013 \\
\hline
\end{tabular}

Nota. En esta tabla se consignan los datos experimentales ya descritos, P representa la parcela a la que pertenece el individuo, $\mathrm{Sp}$, el número asignado a su especie y Num el número individual de cada mangle.

Fuente: Elaboración propia basado en Cortés (2010).

A partir las anteriores tablas es posible observar que en esta zona de muestreo hay una menor cantidad de individuos con respecto a la zona de muestreo Muelle ENAP. Sin embargo, al igual que en la primera zona también se presenta un predominio de la especie Rizophora mangle dada su flexibilidad ecológica que se pone de manifiesto en la ocupación de espacios con diferentes condiciones de salinidad, inundación y tipo de sustrato, situación similar a la encontrada por Cortés (2010) en un estudio realizado en la Bahía de Cispatá Córdoba. 
Tabla 6. Cálculo de IVI en la zona Punta Recluta

\begin{tabular}{|l|l|l|l|l|l|}
\hline \multicolumn{1}{|c|}{ Especie } & \multicolumn{1}{|c|}{$\mathbf{A i} \%$} & \multicolumn{1}{c|}{ Di\% } & \multicolumn{1}{c|}{ Fi\% } & \multicolumn{1}{c|}{ IVI } & \multicolumn{1}{c|}{ IVI\% } \\
\hline Rhizophora mangle & $94,12 \%$ & $93,48 \%$ & $75,00 \%$ & $262,60 \%$ & $87,54 \%$ \\
\hline Laguncularia Racemosa & $5,88 \%$ & $6,52 \%$ & $25,00 \%$ & $37,40 \%$ & $12,46 \%$ \\
\hline
\end{tabular}

Nota. Es claro el predominio ahora del Rhizophora mangle en la zona de muestreo 2, con un IVI\% del $87,54 \%$ representa casi la totalidad de la importancia de la zona, la importancia en función de la Biomasa (Di\%) y la abundancia (Ai\%) refleja la misma conclusión.

Fuente: Elaboración Propia basado en Cortés (2010).

Con base en lo observado en la Tabla 6, el IVI de la especie Rizophora mangle (87,54\%) es muy superior al de la especie Laguncularia racemosa $(12,46 \%)$, resaltando que a diferencia de la Zona 1, en la Zona 2 no se registró presencia de $\mathrm{Co}$ nocarpus erectus, siendo esto un cambio en la estructuración y distribución del bosque de manglar, esto se puede explicar debido a que esta especie es normalmente blanco de acción antrópica para la obtención y uso de madera situación que ocurre frecuentemente en estudios desarrollados en Córdoba (Cortés, 2010), por lo tanto al igual que en la zona 1 el mangle rojo se registra con mayoría de individuos en cada una de las parcelas pertenecientes a este transecto, a diferencia del mangle blanco que solo registró un individuo en la parcela 2, lo que se traduce en que el valor de la Frecuencia Relativa de las dos especies fue diferente para el mangle blanco $25 \%$ y para el mangle rojo $75 \%$, lo que indica que en las tres parcelas quien tiene presencia es el mangle rojo, debido a que la distribución del mangle blanco se limita a la parcela número 2 del transecto; de otro lado la Abundancia Relativa del mangle rojo presentó un valor de $94,12 \%$ el cual está muy por encima del valor para mangle blanco $(5,88 \%)$ en esta zona de muestreo, lo que indica que el número de individuos de mangle rojo es muy superior con respecto al mangle blanco teniendo con relación a la superficie comprendida por cada una de las parcelas; finalmente en el caso de la Dominancia Relativa, el mangle rojo obtuvo un $93,48 \%$ siendo como en el caso de las otras medidas superior al mangle blanco, es decir que la suma de las áreas de los troncos determinadas a la altura del pecho es mayor en el caso del mangle rojo, significando que esta especie al igual que en la zona de muestreo 1, está mejor adaptada a las condiciones actuales del ecosistema en los dos transectos im- plementados como área de estudio constituyendo la mayor biomasa en la zona, el anterior análisis se realizó con base en Curtis y McIntosh (1951) y Cortés (2010).

La estructuración del bosque de mangle encontrado en la isla de Manzanillo es congruente con los explicado por Prahl (1989) quien afirma que los manglares ribereños están compuestos en su mayoría por Rhizophora mangle, Laguncularia Racemosa y Avicennia germinans. Se considera esta clase de mangle como punto de referencia debido a sus condiciones adaptativas le permiten estrategias de colonización. De otro lado se explica la presencia de Conocarpus erectus porque, aunque se determine la predominancia de unas especies, no implica que otras no puedan poblar la zona. Un estudio realizado en la ciudad de Cartagena titulado Mangles de Cartagena de Indias: "Patrimonio Biológico y fuente de biodiversidad" (Díaz, Castro y Manjarrez, 2010) determinó que, en los bosques de mangle presentes en la ciudad, entre las diferentes especies de mangle se encuentra una población de esta especie, lo que indica que, si existen poblaciones de este mangle en la ciudad, sobre todo en zonas estables, más alejadas de la línea de costa, para el caso de la zona muestreada se encontró un claro predominio de la especie Rizophora mangle en los dos transectos como se muestra en las Figuras

Cabe resaltar, que en las dos zonas de muestreo se pudo evidenciar gran cantidad de basuras, en su mayoría desechos plásticos que van desde botellas hasta muebles, cojines, contenedores plásticos de combustibles e incluso juguetes, factor que puede influir en el normal desarrollo y distribución de las especies del bosque de manglar en la Isla de Manzanillo, contribuyendo con la ausencia de especies como es el caso del mangle 
negro (Avicennia germinans) y mangle Zaragoza (Conocarpus erectus para la estación 2) en el área muestreada; es importante mencionar que para el caso de "Punta Recluta" se pudo observar un espécimen de mangle negro, con la madera seca, ubicado en forma horizontal con respecto al suelo y con las raíces expuestas, dicha posición permitió evidenciar neumatóforos en las raíces, este hallazgo se pudo deber a la acción de ráfagas fuertes de viento que en ocasiones azotan la isla; dadas las condiciones del árbol no se reportó dentro de la respectiva parcela. Teniendo en cuenta lo anterior, es pertinente traer a colación que el mangle negro es la especie que se encuentra más alejada de la línea de costa en suelo firme, por lo que es mucho más fácil su acceso por parte del hombre para su sobreexplotación, por su excelente calidad de madera para construcciones artesanales como horcones y carbón (Verarboles, 2018).

Con base en toda la información presentada y analizada, se pudo evidenciar que la distribución de especies de manglar en la isla de Manzanillo es bastante particular, producto de un acentuado efecto antrópico visible a simple vista que repercute directamente en la presencia o ausencia de la especies, situación observada con el cálculo del IVI, en ese contexto las zona de estudio puede tipificarse según el Ministerio de Ambiente (1995) como una zona de recuperación, pues comprenden zonas de manglar que, debido a su mal estado de conservación, impactos altos o en estado de degradación, que requiere acciones encaminadas a recuperar bienes y servicios como primera opción de uso.

\section{Conclusiones}

Una vez realizado el análisis de resultados del presente proyecto, se determinó que las especies de mangle presentes en la isla de Manzanillo son, en gran proporción el mangle rojo, seguido del mangle blanco y en pocas cantidades de individuos de mangle zaragoza.

La distribución de especies de manglar encontrada en el área objeto de estudio, está dada por el efecto antrópico evidenciado y además por la capacidad de las especies reportadas para sobreponerse a la adversidad del medio.

La especie Rhizophora mangle presento el mayor índice IVI en todas las parcelas muestreadas, indicando un alto nivel de resiliencia dadas las condiciones adversas encontradas como la presencia de basuras, en ese orden de ideas en menor medida se encuentran Laguncularia racemosa y Conocarpus erectus.

\section{Referencias bibliográficas}

Calderón, C., Aburto, O., \& Ezcurra, E. (2009). El valor de los manglares. Biodiversitas, (82) 1-6.

Cortés, D. (12 de 11 de 2010). Biblioteca Digital Universidad Nacional de Colombia. Obtenido de http://www.bdigital.unal.edu.co/4153/1/denissevivianacortescastillo.2010.pdf

Curtis, J. T., \& Mcintosh, R. (1951). The inter relation of certain analytic and synthetic phytosociological characters.

Díaz, C., Castro, I., \& Manjarrez, G. (2010). Manglares de Cartagena de Indias: Patrimonio biológico y fuente de biodiversidad. Cartagena: Fundación Universitaria Tecnológico de Comfenalco.

Ecured. (20 de Mayo de 2018). Mangle rojo. Obtenido de https://www.ecured.cu/Mangle_rojo

Escuela Naval de Cadetes "Almirante Padilla". (2016). Portafolio de Investigación, Desarrollo e Innovación. Cartagena: Alpha Editores.

Google. (30 de Mayyo de 2018). Google Earth. Obtenido de https://earth.google.com/web/

Jacome, E. (8 de Abril de 2006). MailxMail. Obtenido de El mangar cartagenero: http://www.mailxmail.com/curso-manglar-cartagenero/conocarpus-erecta-mangle-zaragoza 
Mejía, L., Sanjuan, A., Grijalba, M., \& Niño, L. (2014). Bosque de manglar un ecosistema que debemos cuidar. Cartagena: Alpha editores.

Ministerio de Medio Ambiente. (21 de Diciembre de 1995). Resolución 1602 del 21 de diciembre de 1995. por medio de la cual se dictan medidas para garantizar la sostenibilidad de los manglares en Colombia. Bogotá, Cundinamarca, Colombia.

Olguín , E., Hernández , M., \& Sanchez-Galván, G. (2007). Contaminación de manglares por hidrocarburos y estrategias de biorremediación y fitorremediación y restauración. Revista Internacional de Contaminación Ambiental, 23 (3) 139-154.

Prahl, H. (1989). Manglares de Colombia. Bogotá: Villegas editores.

Quiceno, N., Tangarife, G., \& Alvarez, R. (2015). Estimación contenido de biomasa, fijación de carbono y servicios ambientales en un área de bosque primario en el resguardo indígena Piapoco Chiguiro-Chátare de Barrancomina Departamento de Guainía Colombia. Luna Azul, 171-202.

Ulloa-Delgado, G., Sánchez-Páez, H., Gil-Torres, W., Pino-Rengifo, j., Rodríguez-Cruz, \& Alvarez-Leon, R. (1998). Conservación y uso sostenible de los manglares del caribe Colombiano. Bogotá: Impreandes.

Verarboles. (10 de Mayo de 2018). Verarboles. Obtenido de http://www.verarboles.com/Mangle\%20 Negro/manglenegro.html 